\title{
Research on Resource Management of Phased-Array Radar in Target Tracking
}

\author{
Sun Qing, Zhang Qin, Tao Jianfeng
}

The Air-defense and Anti-missile Institute, Air Force Engineering University. Shannxi Xi'an 710051, China

Keywords: Phased Array Radar; Resource Management; Resource Scheduling; Target Tracking.

Abstract. Scheduling of phased-array radar resource is an urgent problem of its tracking and control. In this paper, optimization control model of phased-array radar has been analyzed and the resource management of a certain type of radar in target tracking has been developed, which will provide theory reference to its battle management.

\section{Introduction}

Target tracking and controlling of phased array radar have been focused widely all the time[1-3]. It can provide a basis for battle management that analyzing the usage of radar resource in the process of target tracking. Once contacting a target in searching course, phase array radar will track it and meanwhile, continue searching all the monitoring airspace. One of the working mode is named tracking while searching(TWS), and another named tracking and searching(TAS).

In order to economize transmitting power and the amount of equipment, the data rate of searching should be relaxed, which means bigger searching interval is permitted. However, the reliability and precision of tracking should be guaranteed to supply the requirements such as multitarget track correlation, and smaller tracking interval is needed. To resolve this contradiction, tracking time should be placed in searching interval, namely TAS. In multi-target tracking, the mode of TAS will give full play to the advantages of phased array radar and the difficulty of which is the management of radar resource, especially to the radar of detecting ballistic target, remotely involve the problems of multi-target tracking, the adaptive adjustment is target moving detection and data rate.. And so, it places greater demands on the resource management of phased array radar that resource scheduling module should allocate resources of time and power to various tasks so as to make full use of the overall efficiency.

From the aspect of electronic intelligence, we should master that how to use the resource of phased array radar to match the corresponding event task and make full use of the modern radar so as to realize the research of its working mode. The system structure of phased array radar is showed in Figure 1.

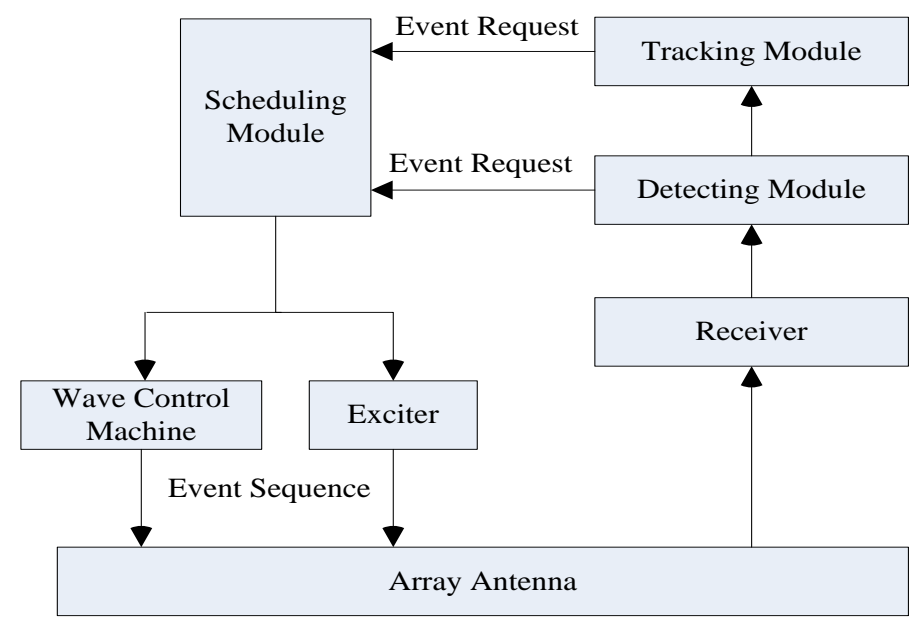

Fig.1 System structure of phased array radar 


\section{Resource scheduling of phased array radar}

The characteristic of phased array radar is the difference between working modes of phased array radar and normal radar. After deterministic design, the working mode and materiel task of mechanical scanning radar are correspondingly solidificated. In practical working, the normal radar will accomplish tasks obey the time rules and its function is simple relatively. As to phased array radar, the usage of working mode is determined by task scheduling. Dividing the burdened tasks into six kind of radar events, and defining their driving priority. According to different radar events, invoke corresponding working mode to finish radar event with conforming the principle of optimized resources disposition and to accomplish multi-functions finally.

Table 1. Static priority of six radar events

\begin{tabular}{ccc}
\hline Task Type & Meaning & Static priority \\
\hline GRB & Guidance & 6 \\
PTRB & Accurate Tracking & 5 \\
LRB & Loss of Tracking & 4 \\
CRB & Confirmation & 3 \\
TRB & Coarse Tracking & 2 \\
SRB & Searching & 1 \\
\hline
\end{tabular}

Phased array radar can work in multi-patterns at the same time, which requires the radar processes modern scheduling models so as to arrange the time and energy resources intelligently, that is the problem of sensors allocation. Sensors constant multiple working modes and working parameters, and so we can realize invoking different working parameters by sensors allocation to implement the aim that the optimal parameters can be allocated to appreciate working mode.

The resource scheduling of phased array radar can be divided into two parts, the priority decision of radar working task and scheduling decision, which is showed in Figure 2.

Related with the allocation of radar sensor-team, the former determines the priority partitioning to accomplish multiple radar tasks. Corresponding to different priorities, different sensor-teams determine the working mode of radar. And the latter arranges executable radar working tasks, which means allocate time and energy according to various working modes.

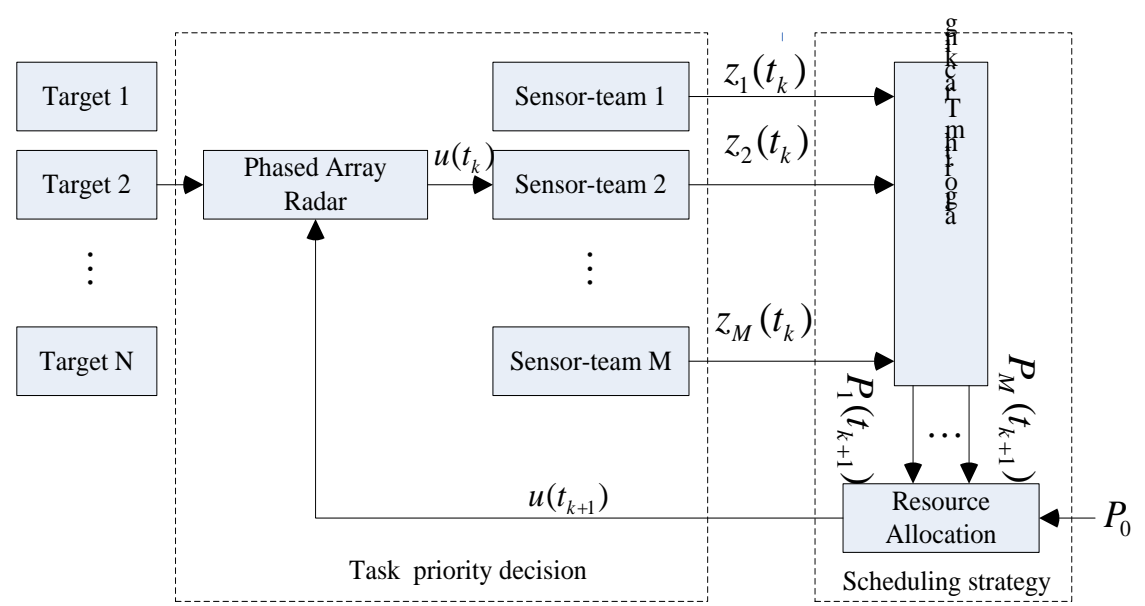

Fig.2 resource scheduling functional block diagram of phased array radar

\section{Scheduling strategy}

Generally, the resource scheduling strategy of phased array radar contains fixed templates, multitemplate, adaptive scheduling and so on. The fixed template scheduling is mostly adopted in modern radar. For fixed radar working sequence, the scheduling will be stable and reliable, however, 
it also has the defects of being inflexible relatively, which will against tracking targets with high speed, long distance and smaller RCS. Adaptive scheduling strategy being more complex and flexible should be adopted in resource management of modern phased array radar.

\section{Scheduling management}

Phased array radar will place completing task first but not unilaterally pursuit precision in multitarget tracking. For example, as to a common target, phased array radar will supply precision of keep tracking. Improve any target tracking accuracy will influence the other target tracking consequentially. However, high accuracy will be adopted in tracking important target or guidance one. For fixed system resource, it's unscientific to analyze management methods from the aspect of number or accuracy unidirectionally. Therefore, various precision control ling methods should be pressed to different targets according to radar tasks.

\section{Analysis to the optimization model of scheduling management}

The strategy based on covariance control is generally used to allocate the resource of phased array radar as to the problems of radar resource management involving the index of tracking accuracy, and the fundamental idea of which is presetting a desired tracking accuracy, named the expected covariance matrix, to every target, and controlling the sensors, making the actual covariance approach the expected one in a certain measurement and criterion, meanwhile, the following system resource will be used to maintain the other target tracking or detecting new. The general optimization model of resource management can be describes as the following form.

Hypothesis the preset expected covariance matrix to D known targets is $P_{0}^{i},(\mathrm{i}=1,2, \ldots, D)$, and the control vector is described as follow:

$$
U\left(t_{k}\right)=\left\{u(t) \mid u(t)=0,1,2, \ldots, D ; t=t_{1}, t_{2}, \ldots, t_{k}\right\}
$$

Here, $u(t)$ expresses the adopted working mode of phased array radar at time of $t, u(t)=0$ describes that radar performs searching task. And $u(t)=j,(j \neq 0)$ describes processing tracking to target $j$. So, the optimization controlling model of resource management can be expressed as formula (2).

$$
\begin{aligned}
& u\left(t_{k+1}\right)=j_{0}\left(t_{k+1}\right) \\
& =\min _{j \in\{0,1, \ldots, D\}} F\left[P_{0}^{i}, P^{i}\left(t_{k+1} \mid U\left(t_{k}\right), u\left(t_{k+1}\right)=j\right), i=1,2, \ldots, D\right]
\end{aligned}
$$

Here, the expression of $P^{i}\left(t_{k+1} \mid U\left(t_{k}\right), u\left(t_{k+1}\right)=j\right)$ means the covariance matrix of tracking error

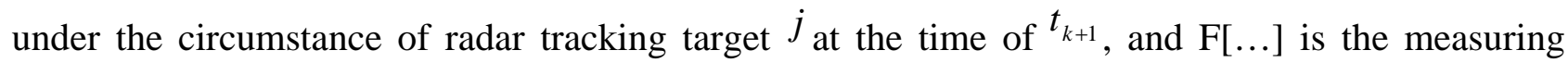
function for calculating the double set matrix deviation. Formula (2) is the criterion for the sensors to choose working mode of the next time point. In order to make all the tracking error covariance matrixes approach the preset ones in a certain measuring criterion, it is defined the minimum value of function $\mathrm{F}[\ldots]$ as the principle of phased array radar controlling.

As to the specific expression form of function F[...], two types of resource management criterion can be proposed. One is the criterion of minimizing the mean deviation, and the other is the criterion of minimizing the deviation to maximum covariance, and we mark them F-1 and F-2 separately.

As to the criterion of F-1, the expression of optimization management model to phased array radar resource is formula (3).

$$
\begin{aligned}
& u\left(t_{k+1}\right)=j_{0}\left(t_{k+1}\right) \\
& =\min _{j \in\{0,1, \ldots, D\}} \frac{1}{D} \sum_{i=1}^{D} f\left[P_{0}^{i}, P^{i}\left(t_{k+1} U\left(t_{k}\right), u\left(t_{k+1}\right)=j\right), i=1,2, \ldots, D\right]
\end{aligned}
$$

And as to the criterion of F-2, the expression is formula (4).

$$
\begin{aligned}
& u\left(t_{k+1}\right)=j_{0}\left(t_{k+1}\right) \\
& =\min _{j \in\{0,1, \ldots, D\}}\left[\max _{i \in\{1, \ldots, D\}} f\left[P_{0}^{i}, P^{i}\left(t_{k+1} \mid U\left(t_{k}\right), u\left(t_{k+1}\right)=j\right), i=1,2, \ldots, D\right]\right]
\end{aligned}
$$


Function $f(A, B)$ describes the diversity measure between matrix $\mathrm{A}$ and $\mathrm{B}$, and which can choose various expressions according to requirements. Many measuring methods can be used to compare the variance between the actual covariance matrix and the expected one, such as the common used 2-matrix norm, the column norm, Row norm, Frobenius-norm, matrix trace, determinant and the singular value decomposition of matrix.

The general optimization model of resource management mentioned above is not limited to the number of tracking-target or the state estimating dimension of every target in an environment. It is because that the corn of this optimization model is allocating radar system resource by a chosen measuring criterion according to the preset expected covariance matrix. Once the tracking state and the expected covariance index of current sensors are given, the resource allocating form of phased array radar in next time point can be obtained.

Fig.2 gives out the whole operation flow of phased array radar system in tracking mode. Firstly, radar system will process data acquisition of targets in external environment according to the controlled quantity $u\left(t_{k}\right)$, and transmit the measuring data to data processing unit to operate tracking correlation, filtering and prediction. And then, according to the processing result from tracking algorithm and the expected covariance $P_{0}$ preset by the system, the resource management module will determine the controlled quantity $u\left(t_{k+1}\right)$ to phased array radar in the next time point, and finally, transmit which to radar system so as to control data acquisition.

Hypothesis that there has D targets in surveillance area. The task of phased array radar is maintaining tracking $\mathrm{D}$ targets and meanwhile detecting new ones. The expected covariance matrixes preset for these $\mathrm{D}$ targets can be expressed as $P_{0}^{i},(\mathrm{i}=1,2, \ldots, D)$. We hypothesis that the update mode of the closet filter before time point $t_{k}$ to these D targets are $\left\{t_{k(i)}, P^{i}\left(t_{k(i)}\right)\right\},\left(t_{k(i)} \leq t_{k}\right)$, and here the subscript $k(i)$ expresses discrete number of the $k$-th Kalman filter to target $i$.

The discrete state equation of target $i$ is as formula (5).

$$
X^{i}\left(t_{k+1}\right)=F^{i}\left(T_{k}^{i}\right) x^{i}\left(t_{k(i)}\right)+G^{i}\left(T_{k}^{i}\right) w^{i}\left(t_{k(i)}\right)
$$

Here, $X^{i}\left(t_{k(i)}\right) \in \mathfrak{R}^{n}$ expresses the state vector of target $i$ at time point $t_{k(i)}, w^{i}\left(t_{k(i)}\right) \in \mathfrak{R}^{n}$ is the white noise vector of this system, $Q^{i}\left(t_{k(i)}\right)$ is the covariance matrix, $F^{i}\left(T_{k}^{i}\right)$ is the state transition matrix and $G^{i}\left(T_{k}^{i}\right) \in \mathfrak{R}^{n \times r}$ is the input matrix of time point $t_{k}$, and $T_{k}^{i}=t_{k+1}-t_{k(i)}(k=0,1, \ldots$, expresses target sampling interval at time point $t_{k+1}$

The measurement equation of radar system is as follows.

$$
z^{i}\left(t_{k}\right)=H^{i} x^{i}\left(t_{k}\right)+v^{i}\left(t_{k}\right)
$$

Here, $z^{i}\left(t_{k}\right) \in \mathfrak{R}^{n}$ expresses the measurement vector to target $i$ at the time point $t_{k}, v^{i}\left(t_{k}\right) \in \mathfrak{R}^{n}$ is measurement noise. The covariance matrix is $R^{i}\left(t_{k}\right)$ and $H^{i} \in \mathfrak{R}^{n}$ is the observation matrix. Noise $v^{i}(. .$.$) and w^{i}(. .$.$) are statistical independence, and irrelevant with initial state.$

Prediction covariance matrix and filter covariance in Kalman filtering equation are separately showed in formula (7) and formula (8).

$$
\begin{gathered}
P^{i}\left(t_{k+1}^{-}\right)=F^{i}\left(T_{k}^{i}\right) P^{i}\left(t_{k(i)}\right) F^{i T}\left(T_{k}^{i}\right)+G^{i}\left(T_{k}^{i}\right) Q^{i}\left(t_{k(i)}\right) G^{i T}\left(T_{k}^{i}\right) \\
P^{i}\left(t_{k+1}\right)=\left[I_{n}-K^{i}\left(t_{k+1}\right) H^{i}\right] P^{i}\left(t_{k+1}^{-}\right)
\end{gathered}
$$

Here, $K^{i}\left(t_{k+1}\right)$ expresses the gain of Kalman filtering.

Set all controlled vectors of phased array radar before time point $t_{k}$, and the expression will be $U\left(t_{k}\right)=\left\{u(t) u(t)=0,1,2, \ldots, D ; t=t_{1}, t_{2}, \ldots, t_{k}\right\}$.

Assume controlled vector $u\left(t_{k+1}\right)$ at time point $t_{k+1}$ is $u(k+1)=j, j \in(0,1, \ldots, D)$, and the tracking error covariance matrix of every target at time point $t_{k+1}$ can be describes as formula (8). 


$$
P^{i}\left(t_{k+1} \mid U\left(t_{k}\right), u\left(t_{k+1}\right)=j\right)=\left\{\begin{array}{l}
P^{i}\left(t_{k+1}^{-}\right), i \neq j \\
P^{i}\left(t_{k+1}\right), i=j
\end{array}\right.
$$

From formula(9) we can obtain the error covariance matrix of all targets at time point $t_{k+1}$, and substituting which into resource management model, the resource allocation result of phased array radar at every time point can be introduced.

Integrated the above analyses it is known that at time point $t_{k+1}$, the calculating results of actual target covariance matrix $P^{i}\left(t_{k+1} \mid U\left(t_{k}\right), u\left(t_{k+1}\right)=j\right)$ will be different when phased array radar processes tracking various targets or searching new ones. Based on formula (3) and formula (4), resource management algorithm will control radar system to make the actual covariance matrix of target approach the expected one best in a certain sense.

We process estimation work to the resource occupation to a certain type of phased array radar in target tracking.

From the analysis to the course of common target tracking we can see that the data rates of rough tracking and accurate tracking are separately $1 \mathrm{~Hz}$ and $5 \mathrm{~Hz}$. Considering tracking ten batches targets, and if the beam resident period is $6 \mathrm{~ms}$, the resource occupation rate will be $6 \%$ in rough tracking and $30 \%$ in accurate tracking.

As to ballistic targets, the data rate is $10 \mathrm{~Hz}$. If the beam resident period is $6 \mathrm{~ms}$, the resource occupation rate of every ballistic target channel will be $6 \%$. If tracking with double backup channels is adopted, and double channels are prepared for separated targets detection, and the resource occupation rate will be $30 \%$.

In addition, intercepting missile should be launched to attack aerial target. The intercepting missile can process single channel tracking by acknowledgement signal and the data rate should be coincident with ballistic target, which means the occupation rate of every intercepting missile is $6 \%$. If two intercepting missiles are launched to attack target, the resource occupation will be $12 \%$.

In summary, if all the tracking channels work, the system resource occupation will be $72 \%$, and the rest time can be used to process identification or supervision important targets.

\section{Conclusion}

It is an important research subject that realize significant scheduling to multi-function phased array radar and optimization to system resource in the complex environment with multi-target tracking and detecting. In this paper, the resource management of phased array radar in targettracking has been detailed analyzed and according to a certain type of radar, the resource occupation has been estimated in target tracking. Actually, in further research, we should synthetically consider the resources of time, power, computer and even radar hardware to constraint the scheduling algorithm.

\section{References}

[1]Luo Qun, Zhou Wanxing, Ma Lin. The World Ground Radar Hangbook [M]. Beijing:National Defence Industry Press,2005.

[2]Zhang Guangyi. Phased Array Radar system[M].Beijing:National Defence Industry Press,1994.

[3] ZHAO Yan li, LIU Jian, LUO Peng fei. Adaptive Sample Target Tracking Algorithm for Phased Array Radar [J].Modern Radar. 2003, 25(5): 37-39.

[4] Wang Jing. System Simulation of Phased Array Radar [ J]. Experiment Science \& Technology, 2006, 4(3): 118-121.

[5] Jiang Xumin. Multiple Task Management of Phased Array Radar [J].Electronic Science and Technology,2008. Vol.21(6).

[6] ZENG Guang, LU Jian bin, HU Wei dong. Research on Adaptive Scheduling Algorithm for Multifunction Phased Array Radar[J]. Modern Radar, 2004, 26(6). 
[7]Rec. ITU R P. 618-8. Propagation data and prediction methods required for the design of Earthspace telecommunication systems, 2003.

[8]WANG Kai, ZHANG Hui. Development Demand and Direction of Shore-Based Multifunctional and Phased Array Radar[J].Electronic Engineering, 2011,7(4):31-34.

[9]ZHOU Wen-hui. Research on R esource Management Technology for Phased Array Radar and Its Network in Tracking System[D].Changsha:National University of Defense Technology,2004.

[10]MAO Yi-na.Resource Management under Tracking Mode and Task Scheduling for Phased Array Radar[D].Xi'an:University of Electronic Science and Technologyof Xi'an,2011.

[11] QIN Ya-ping, WANG Jian, ZHANG Yu-xi. An Adaptive Data $R$ ate Target Tracking Algorithm of Phased-Array R adars[J]. R adar \& Ecm,2011. 